Revista Baiana de Saúde Pública

ARTIGO ORIGINAL

\title{
FARMÁCIA MAGISTRAL: SUA IMPORTÂNCIA E SEU PERFIL DE QUALIDADE
}

\author{
Rudy Bonfilio \\ Guilherme Luz Emerick ${ }^{b}$ \\ Antônio Netto Júnior ${ }^{c}$ \\ Hérida Regina Nunes Salgado ${ }^{a}$
}

\begin{abstract}
Resumo
O setor magistral representa um importante segmento do mercado brasileiro de medicamentos. Entretanto, a qualidade dos produtos fornecidos pelos estabelecimentos é frequentemente discutida e a Agência Nacional de Vigilância Sanitária (ANVISA) tem demonstrado uma preocupação com os produtos magistrais, pela promoção de consultas públicas abordando o assunto e pelo aumento do rigor nas legislações específicas. Assim sendo, as farmácias magistrais desempenham um importante papel no contexto da Política Nacional de Medicamentos, que objetiva garantir a promoção do uso racional e o acesso da população a medicamentos essenciais. Tendo em vista a importância da abordagem da qualidade dos produtos manipulados, este artigo apresenta relevantes informações sobre o perfil de qualidade do serviço de saúde prestado por esse setor, mediante a revisão da legislação e de trabalhos científicos que englobam o assunto.
\end{abstract}

Palavras-chave: Setor magistral. Produtos farmacêuticos manipulados. Controle de Qualidade de fármacos e medicamentos. Política Nacional de Medicamentos.

\section{MAGISTRAL PHARMACIES: IMPORTANCE AND QUALITY PROFILE}

\begin{abstract}
The magistral sector represents a significant segment in the Brazilian pharmaceutical market. However, the quality of magistral formulations is often questioned and the National Agency of Sanitary Vigilance (ANVISA) has demonstrated a concern regarding these products, which has been verified in many public consultations approaching this subject and in new legislation aiming to improve public health. Thus, magistral pharmacies play an important role in the context of the National Medicine Politics, which aim to assure promotion of rational use and population access to essential medicines. Due to the paramount importance of the magistral formulation quality,

\footnotetext{
a Departamento de Fármacos e Medicamentos, Faculdade de Ciências Farmacêuticas, Universidade Estadual Paulista (UNESP).

${ }^{b}$ Departamento de Princípios Ativos Naturais e Toxicologia, Faculdade de Ciências Farmacêuticas, Universidade Estadual Paulista (UNESP).

c Programa de Pós-Graduação em Educação Escolar, Faculdade de Ciências e Letras de Araraquara, Universidade Estadual Paulista (UNESP).

Endereço para correspondência: Rudy Bonfilio. Departamento de Fármacos e Medicamentos, Rodovia Araraquara-Jaú, KM 1, Araraquara, São Paulo, Brasil. CEP: 14801-902.rudybonfilio@yahoo.com.br
} 
this article presents information about the basic importance and the quality profile of these products, through the revision of the legislation and of scientific works that approach the subject.

Key words: Magistral sector. Magistral formulations. Pharmaceutical quality control. National Medicine Politics.

\section{FARMACIA MAGISTRAL: SU IMPORTANCIA Y SU PERFIL DE CALIDAD}

\section{Resumen}

El sector magistral representa un importante segmento del mercado brasileño de medicinas. Sin embargo, la calidad de los productos ofrecidos por estos establecimientos es frecuentemente discutida y la Agencia Nacional de Vigilância Sanitária (ANVISA), ha demostrado preocupación con los productos magistrales, por la promoción de consultas públicas abordando el asunto y por el aumento del rigor en las legislaciones específicas. Siendo así, las farmacias magistrales desempeñan importante rol en el contexto de la política Nacional de Medicinas, que busca garantizar la promoción del uso racional y el acceso de la población a las medicinas esenciales. Considerando la importancia del abordaje sobre la calidad de los productos manipulados, este artículo presenta relevantes informaciones sobre el perfil de la calidad del servicio de la salud ofrecido por ese sector, a través de la revisión de la legislación y de trabajos científicos que engloban el asunto.

Palabras-clave: Sector marginal. Productos farmacéuticos manipulados. Control de calidad de fármacos y medicinas. Política Nacional de Medicinas.

\section{INTRODUÇÃO}

As farmácias magistrais representam significativa parcela do mercado brasileiro de medicamentos. Este setor ressurgiu no Brasil no final da década de 1980, após seu desaparecimento quase completo devido ao advento da indústria farmacêutica na década de 1950. ${ }^{1}$ No início eram poucos estabelecimentos voltados principalmente à dermatologia ou à homeopatia, com foco na individualização da prescrição. Com a entrada dos medicamentos genéricos no mercado, o segmento passou a manipular inúmeros medicamentos cujas apresentações são disponibilizadas pela indústria farmacêutica. ${ }^{2}$

O aumento do número de medicamentos manipulados no Brasil resultou em maior preocupação com a qualidade destes produtos. A Agência Nacional de Vigilância Sanitária (ANVISA) publicou, em 19 de abril de 2000, a primeira regulamentação específica para este setor, a RDC № 33. ${ }^{3}$ Esta resolução instituiu as boas práticas de manipulação em farmácias e propiciou significativas evoluções quanto à qualidade dos produtos magistrais. 
Revista Baiana de Saúde Pública

Seguindo o desenvolvimento científico-tecnológico, que demanda padrões de qualidade cada vez mais exigentes, a RDC № 33 passou por modificações, com o intuito de disseminar o conceito de qualidade nos estabelecimentos magistrais, objetivo que não foi alcançado de imediato. Em 2003, devido a alguns acontecimentos relacionados à intoxicação de pacientes por medicamentos manipulados, foi estabelecida a RDC № $354,{ }^{4}$ a qual estabelece critérios específicos para a manipulação magistral de substâncias de baixo índice terapêutico.

Em 2005, a ANVISA lançou a consulta pública número 3, na qual foram discutidos pontos falhos na RDC № 33, com a intenção de estabelecer critérios mais rígidos para a manipulação no Brasil. ${ }^{5}$ Foi então criada a RDC № 214, em 12 de dezembro de 2006, ${ }^{6}$ posteriormente revogada pela RDC NN$^{\circ} 67$, de 8 de outubro de 2007, 7 a qual se encontra em vigor.

A exigência cada vez maior das autoridades sanitárias evidencia a preocupação com a qualidade dos produtos manipulados. O principal ponto que suscita polêmica é o fato de que os estabelecimentos magistrais detêm menor quantidade de recursos financeiros em relação à indústria farmacêutica e isto levanta uma importante questão: os produtos magistrais conseguem manter o mesmo padrão de qualidade dos produtos industrializados?

Objetivando fornecer subsídios para responder a esta questão e promover maior compreensão sobre o papel das farmácias magistrais, este artigo suscita informações relevantes sobre a importância e a qualidade dos produtos manipulados nas farmácias brasileiras.

\section{IMPORTÂNCIA DA FARMÁCIA MAGISTRAL}

A busca pela cura é um gesto instintivo e as mais antigas fontes escritas médico-farmacêuticas são as tabuinhas de argila, provenientes das civilizações da mesopotâmia, e os papiros, provenientes do Egito. Entre os papiros, o mais célebre é o de Ébers, descoberto há cerca de um século, remontado a 1700 a.C. ${ }^{8}$ Por volta do século IV a.C., Hipócrates, médico de Cós, pequena ilha do litoral helênico, introduz a farmácia e a medicina científica. ${ }^{9}$

O termo Farmácia Galênica expressa uma homenagem a Claudius Galenus, médico farmacêutico que viveu em Roma durante o segundo século desta era. Espírito verdadeiramente enciclopédico, sintetizou os conhecimentos farmacêuticos adquiridos até então, atribuindo-Ihes também a concepção de várias formas farmacêuticas. Seus escritos ficaram célebres e muitas formas por ele idealizadas chegaram até os dias atuais. ${ }^{10}$

No fim do século XIX e começo do XX, o conhecimento adquirido ao longo de milênios passa a ser utilizado pelo setor industrial, e o aperfeiçoamento mecânico possibilita novas formas farmacêuticas (comprimidos, drágeas) ou aperfeiçoa as existentes (ampolas, pós, dispersões, pomadas). O surto industrial conduz à difusão do medicamento industrializado 
que, aos poucos, conquista a preferência do médico e dos pacientes, embora favorecendo a automedicação. Com isso, reduz-se o aviamento do receituário e a manipulação na farmácia oficina, que se transforma aos poucos em mero estabelecimento comercial, dispensador de medicamentos preparados em série. ${ }^{8}$

O setor farmacêutico, porém, possui um propósito mais amplo, objetivando a prestação de um serviço de qualidade no setor de saúde. A Resolução № . 357, de 20 de abril de 2001, do Conselho Federal de Farmácia (CFF), ${ }^{11}$ define a farmácia como um estabelecimento de prestação de serviços farmacêuticos de interesse público e/ou privado articulada ao Sistema Único de Saúde (SUS), destinada a prestar assistência farmacêutica e orientação sanitária individual ou coletiva, onde se processe a manipulação e/ou dispensação de produtos e correlatos com finalidade profilática, curativa, paliativa, estética ou para fins de diagnósticos.

A manipulação necessita de seleção criteriosa de princípios ativos e da dose para obter a eficácia terapêutica desejada. Além disso, a farmácia magistral busca trabalhar em parceria com os médicos, ao oferecer informações pertinentes de cada fármaco. A fórmula magistral valoriza o médico que a prescreve, melhora a relação médico-paciente e permite um equilíbrio da fórmula para o paciente que, como pessoa única e individual em sua sintomatologia, nem sempre se adapta a formulações já estabelecidas. ${ }^{12}$

A Política Nacional de Medicamentos (PNM), parte integrante da Política Nacional de Saúde aprovada pela Comissão Intergestores e pelo Conselho Nacional de Saúde, tem como objetivo garantir a necessária segurança, eficácia e qualidade dos medicamentos, a promoção de seu uso racional e o acesso da população àqueles considerados essenciais. ${ }^{13}$

Neste contexto, insere-se o conceito do Uso Racional de Medicamentos, que é definido como processo que compreende a prescrição apropriada; a disponibilidade oportuna e a preços acessíveis; a dispensação em condições adequadas; e o consumo nas doses indicadas, nos intervalos definidos e no período de tempo indicado de medicamentos eficazes, seguros e de qualidade. A análise deste conceito permite verificar-se que, sem as formulações magistrais, seria impossível alcançar-se o uso racional de medicamentos, pois nem sempre os preços dos medicamentos industriais são acessíveis à população de baixa renda.

Estudo demonstrou que o segmento da farmácia magistral apresenta uma viabilidade financeira superior para o usuário, quando comparado com os demais setores farmacêuticos. ${ }^{14}$ O objeto de estudo foi o preço de venda de medicamentos manipulados, referência, genérico e similar. Demonstrou-se a enorme diferença entre se comprar um medicamento manipulado e um industrializado. Para um determinado produto manipulado foi encontrado um preço médio 
Revista Baiana de Saúde Pública

de venda de $R \$ 13,33$, enquanto para o medicamento referência, genérico e similar foram encontrados preços médios de venda de $R \$ 63,25, R \$ 25,04$ e $R \$ 25,71$, respectivamente.

Conforme dados da Associação Nacional das Farmácias Magistrais (ANFARMAG), a farmácia magistral representa cerca de $10 \%$ de todo o mercado de medicamentos no Brasil. Os medicamentos manipulados são economicamente mais vantajosos do que os de referência, genéricos ou similares. Foi constatado, entre os menores preços cotados, que todos estavam acima do menor preço dos manipulados, ocorrendo uma variação superior a 530\% do medicamento referência, $189 \%$ do medicamento genérico e $173 \%$ de outros medicamentos similares. No Brasil, são mais de 5.500 estabelecimentos que contribuem para o desenvolvimento econômico com mais de 60 mil empregos diretos e 240 mil indiretos, movimentando em toda a cadeia cerca de 1 bilhão de dólares por ano. ${ }^{15}$ Contudo, a importância da farmácia magistral não se restringe a questões econômicas. Este segmento tem a constante preocupação de fornecer medicamentos de alta qualidade, com o intuito de valorizar o atendimento médico/paciente.

Além disso, há a necessidade da presença constante do profissional farmacêutico, que pode exercer as seguintes funções: manipular e dispensar fórmulas alopáticas e homeopáticas; dispensar e fracionar plantas de aplicações terapêuticas e medicamentos fitoterápicos, observados o acondicionamento adequado e a classificação botânica; dispensar drogas (matérias-primas), insumos farmacêuticos (matérias-primas aditivas), correlatos e alimentos para fins especiais; prestar serviços farmacêuticos de acordo com a legislação sanitária; promover ações de informação e educação sanitária e prestar serviço de aplicação de injeção. Desta forma, o paciente pode obter informações precisas sobre a prescrição médica, além de tirar quaisquer dúvidas a respeito do medicamento manipulado.

Enfim, não se deve esquecer a importância de um estabelecimento de saúde como a farmácia. Para reforçar o papel das farmácias, entidades farmacêuticas e organizações de saúde de todo o país uniram-se para a aprovação, na Câmara dos Deputados, do Projeto de Lei 4.385/1994. O projeto dispõe sobre ações e serviços de assistência farmacêutica e propõe transformar as farmácias em unidades de prestação de serviços de saúde. A busca desenfreada pelo lucro, baseada em práticas comerciais abusivas, não se pode sobrepor aos preceitos éticos e técnicos que a atividade requer. Os farmacêuticos devem sempre prezar pela qualidade dos produtos e das informações técnico-científicas prestadas aos clientes, para que a credibilidade das farmácias seja restabelecida.

\section{QUALIDADE DOS MEDICAMENTOS NA FARMÁCIA DE MANIPULAÇÃO}

A ANVISA exige altos padrões de qualidade dos medicamentos produzidos em escala industrial. Por outro lado, a preocupação com os medicamentos manipulados levou à 


\title{
PROGRAMA NACIONAL DE ELIMINAÇÃO DA HANSENÍASE: UM ESTUDO SOBRE A AVALIABILIDADE DO PROGRAMA E DAS SUAS AÇÕES EM ÂMBITO ESTADUAL E MUNICIPAL
}

\author{
Victor Hugo Maia Valois Costa ${ }^{a}$ \\ Larissa de Amorim Cavalcantia \\ José Antônio Diniz Faria-Junior ${ }^{a}$ \\ Emy Guerra Kitaoka \\ Gisele de Sá Mascarenhas ${ }^{a}$ \\ Nildo Batista Mascarenhas ${ }^{b}$ \\ Camila de Andrade Nascimento ${ }^{a}$ \\ Renata Barbosa Paolilo \\ Fernanda Mota Ramos ${ }^{b}$ \\ Paula Myllane F. dos Santos Silva
}

\begin{abstract}
Resumo
A hanseníase é uma doença infecciosa, de evolução crônica, causada pelo Mycobacterium leprae. É uma enfermidade estigmatizante e tem um passado de discriminação e isolamento dos doentes. O presente estudo objetiva verificar a avaliabilidade do Programa Nacional de Eliminação da Hanseníase para uma futura avaliação sistemática. A coleta de dados foi feita por meio de entrevistas com informantes-chaves, nas esferas estadual e municipal de governo, e observação sistemática in loco, para posterior comparação com o preconizado pelo plano selecionado. Houve grandes diferenças na definição do Programa e de seus objetivos por parte dos gestores. Há reuniões avaliativas em quase todos os níveis do sistema; as informações quanto ao controle dos comunicantes e a adesão ao tratamento, entretanto, ainda são discordantes. Os recursos estão sendo repassados como o previsto pela legislação brasileira, mas a falta de uma verba destinada exclusivamente para o controle da hanseníase dificulta seu manejo. Evidenciou-se que treinamentos para os profissionais de saúde são realizados, no entanto eles ainda parecem insuficientes. Concluiu-se que o Programa Nacional de Eliminação da Hanseníase é avaliável.
\end{abstract}

Palavras-chave: Hanseníase. Programa. Eliminação.

a Faculdade de Medicina da Universidade Federal da Bahia (UFBA). Estudantes de graduação de medicina.

b Escola de Enfermagem da Universidade Federal da Bahia (UFBA). Estudantes de graduação de enfermagem. Endereço para correspondência: Victor Hugo Maia Valois Costa. Rua Marechal Floriano, n 106, apto. 702, Canela, Salvador, Bahia. CEP: 40110-010. victorhugovalois@hotmail.com 
adoção de medidas pela ANVISA que resultaram no aumento do rigor com relação aos produtos magistrais. Essas medidas, somadas ao acesso cada vez maior à informação e ao grande arsenal terapêutico atualmente disponível, exercem influência nos profissionais de saúde e no paciente, gerando dúvidas frequentes quanto à qualidade dos produtos a serem utilizados na terapia medicamentosa.

Os prescritores contam com diversas fontes de informação sobre os medicamentos, as quais servem como base para o estabelecimento de práticas terapêuticas. É sabido que a informação disponível sobre os medicamentos industrializados é disseminada, em grande medida, pelos próprios fabricantes. ${ }^{13}$ Estes lançam mão de diversos meios para efetuar a publicidade de seus produtos, como financiamento de programas de educação, de associações profissionais ou de revistas médicas; relacionamento com autoridades sanitárias, políticos, professores e especialistas; anúncios em revistas médicas; propagandistas; e a distribuição de amostras grátis, de materiais impressos e de brindes. ${ }^{16}$

Dentro do cenário político-econômico atual, a sociedade moderna apresenta uma tendência ao consumismo, impulsionada pela política de marketing desenvolvida pelas indústrias. Essa política, no setor farmacêutico, pode levar a uma tendência de prescrição de um determinado produto, influenciada pelas fontes de informação. Há estudos que demonstram uma relação direta entre os medicamentos mais anunciados e a frequência de sua prescrição. ${ }^{17}$ Considerando o impacto da propaganda terapêutica, deve-se esperar que o material utilizado seja imparcial e de boa qualidade, o que não ocorre na maioria dos casos. Diversos estudos demonstram que a qualidade da propaganda de medicamentos não atinge um padrão necessário. ${ }^{16,18-21}$

Recentemente, a ANVISA publicou a RDC № 96, ${ }^{22}$ que dispõe sobre a publicidade de medicamentos. Esta resolução expressa claramente que as empresas não podem outorgar, oferecer, prometer ou distribuir brindes, benefícios e vantagens aos profissionais prescritores ou dispensadores, aos que exerçam atividade de venda direta ao consumidor, bem como ao público em geral. Além desta medida, essa resolução traz outros benefícios à população, pois também determina que as informações devam ser comprovadas cientificamente. Essa resolução tem um papel fundamental na promoção de uma melhoria na qualidade das informações sobre os medicamentos industrializados, tanto aos profissionais de saúde quanto à população, que desde então passam a contar com informações mais seguras sobre a qualidade dos medicamentos.

Em contraste com o setor industrial farmacêutico, as farmácias magistrais não possuem os recursos sofisticados de divulgação sobre os produtos, o que ocasiona uma valorização do produto farmacêutico industrializado em detrimento do produto manipulado. 
Revista Baiana de Saúde Pública

Uma ferramenta indispensável na qualidade de medicamentos é o controle de qualidade, que apresenta um alto custo, necessidade de adequação de área física, aquisição de equipamentos e treinamento contínuo de pessoas. ${ }^{23}$ Estas características dificultam a execução do controle de qualidade nas farmácias de manipulação, que em muitos casos não dispõem de recursos suficientes para a execução de tais onerosas práticas, o que leva a um questionamento a respeito da qualidade dos produtos manipulados.

Os requisitos mínimos para a manipulação de medicamentos, porém, são estabelecidos pela RDC N ${ }^{\circ} 67,7$ que abrange questões relacionadas às instalações, equipamentos, recursos humanos, aquisição e controle de qualidade da matéria-prima. Traz ainda as exigências para armazenamento, avaliação farmacêutica da prescrição, manipulação, fracionamento, conservação, transporte, dispensação das formulações e atenção farmacêutica aos usuários.

Com relação ao controle de qualidade dos produtos manipulados, a farmácia tem por obrigação submeter todas as matérias-primas, e por amostragem os produtos acabados, aos testes exigidos. A RDC N ${ }^{\circ} 67$ exige a realização de inúmeras análises. Estas dependem do tipo de forma farmacêutica e exigem os seguintes testes: caracteres organolépticos; solubilidade; $\mathrm{pH}$; peso; volume; ponto de fusão; densidade; avaliação do laudo de análise do fabricante/fornecedor; peso médio; desintegração; grau ou teor alcoólico; volume; viscosidade; teor do princípio ativo; dissolução e pureza microbiológica. ${ }^{7}$ A ANVISA estabelece ainda que as matérias-primas devem vir acompanhadas dos respectivos Certificados de Análise encaminhados pelo fornecedor. Além disso, testes físico-químicos e microbiológicos devem ser realizados para monitorar a qualidade da água de abastecimento, mantendo-se os respectivos registros.

Uma consideração importante é que a farmácia pode decidir realizar ou terceirizar os testes mediante contrato mutuamente acordado e controlado entre as partes, de modo a evitar equívocos na análise de qualidade. A empresa responsável por realizá-los deve estar tecnicamente capacitada para esse fim. ${ }^{7}$

Com base nessas considerações, verifica-se que existe um órgão competente e que há legislações específicas para assegurar a qualidade dos produtos manipulados. Além disso, a legislação vigente é flexível quanto à escassez de recursos para o controle de qualidade nas farmácias magistrais e permite a terceirização desses serviços. Convém ressaltar que existem laboratórios habilitados pela ANVISA, os quais fazem parte da Rede Brasileira de Laboratórios Analíticos em Saúde (REBLAS). Estes laboratórios prestam serviços de elevada confiabilidade dos resultados analíticos, atendendo aos princípios fundamentais de gestão da qualidade analítica e Boas Práticas de Laboratório. ${ }^{24}$ 
Outro fator que merece atenção é o registro de medicamentos. Os produtos registrados a partir de 2003 são divididos em novos, similares, genéricos, fitoterápicos, homeopáticos, biológicos e específicos. ${ }^{25}$ Os medicamentos novos, sejam eles sintéticos ou biológicos, são lançados no mercado com base em estudos que comprovem sua eficácia clínica e sua reduzida toxicidade.

Os produtos similares e genéricos, depois de expirado o prazo de proteção patentária, podem ser industrializados com a mesma formulação do medicamento referência, desde que sejam comprovadas sua equivalência farmacêutica e sua bioequivalência em relação ao produto referência. Essa comprovação é feita por ensaios in vitro e in vivo, os quais asseguram que os produtos similares e genéricos irão produzir o mesmo efeito terapêutico dos produtos já existentes.

Tais estudos não são exigidos das farmácias de manipulação, o que pode ser apontado como um fator preponderante na determinação da qualidade de produtos manipulados. A ANVISA, porém, define equivalentes farmacêuticos, como medicamentos que contêm o mesmo fármaco, na mesma quantidade e forma farmacêutica, podendo ou não conter excipientes idênticos. ${ }^{26}$ É importante salientar, dentro deste conceito, que os produtos para serem equivalentes devem apresentar-se na mesma forma farmacêutica. O papel da farmácia de manipulação, porém, não é produzir cópias dos produtos industrializados. A farmácia magistral, como em muitos países, deve assumir um papel complementar às indústrias, manipulando medicamentos que não são industrializados, em concentrações ou formas farmacêuticas adequadas ao paciente cujo estado clínico exige medicação que foge à padronização. ${ }^{25}$

Outra questão que suscita polêmica é o preço inferior dos produtos manipulados devido aos menores gastos das farmácias magistrais com pesquisa e controle de qualidade. Entretanto, como já apontado, grande parte do custo dos medicamentos industrializados deve-se ao gasto da indústria farmacêutica com publicidade e propaganda. . $^{16,18-20}$

O ponto mais relevante com relação à manipulação de medicamentos é a responsabilidade do profissional farmacêutico na atividade de manipulação, pois um erro na preparação do produto magistral pode ser fatal. A prática da manipulação de medicamentos no país, principalmente os de baixo índice terapêutico, como digitálicos, psicotrópicos e hormônios, entre outros, é uma situação na qual o risco potencial à saúde se apresenta de maneira preocupante, considerando a segurança do usuário. ${ }^{27}$ Foram descritos casos de intoxicação medicamentosa e reações adversas por digitálicos, benzodiazepínicos, levotiroxina, clonidina, colchicina, entre outros. Alguns destes casos evoluíram para coma e óbito, especialmente em crianças. ${ }^{27}$ 
Revista Baiana de Saúde Pública

A RDC № 67, no Anexo II, 7 fixa os requisitos mínimos para a manipulação de substâncias de baixo índice terapêutico. O Anexo III determina as boas práticas de manipulação de hormônios, antibióticos, citostáticos e substâncias sujeitas a controle especial. O Anexo IV trata das boas práticas de manipulação de produtos estéreis. Essas medidas evidenciam uma preocupação com a qualidade destes produtos, mas por si só não garantem que há segurança em sua manipulação, uma vez que a fiscalização deve ser efetiva para obrigar, com rigor, que os estabelecimentos atendam aos requisitos mínimos exigidos pela legislação.

Os erros cometidos em áreas de saúde podem ocorrer em qualquer segmento. Mesmo na indústria farmacêutica, em que a validação de processos e o controle de qualidade de cada lote produzido permitem uma avaliação contínua da qualidade dos produtos, há relatos frequentes de falsificação e de retiradas do mercado. Além disso, no caso de um produto industrializado, um problema na qualidade pode atingir um elevado número de pacientes.

Este trabalho apresentou o que se julga como relevantes informações a respeito da importância da farmácia magistral na prestação de serviços à saúde. As autoridades sanitárias, por meio de órgãos competentes, têm aumentado o rigor com as fórmulas magistrais, assegurando, dessa forma, maior padrão de qualidade desses produtos.

A saúde é um direito constitucional; por isto o farmacêutico, pela manipulação de medicamentos, desempenha um importante papel na disponibilização de um serviço acessível a um maior número de pessoas.

A análise da segurança e qualidade das fórmulas farmacêuticas magistrais não deve ser realizada sob a ótica mercadológica, que em muitos casos prioriza o comércio sustentado em bases econômicas.

Finalmente, a manipulação é uma prática que exige grande responsabilidade e profundo conhecimento dos profissionais de saúde envolvidos, sendo necessário o constante aperfeiçoamento e fiscalização atuante, a fim de se evitar eventuais problemas, os quais são passíveis de ocorrer em qualquer ramo do setor de saúde.

\section{AGRADECIMENTOS}

À Fundação de Amparo à Pesquisa do Estado de São Paulo (FAPESP), ao Conselho Nacional de Desenvolvimento Científico e Tecnológico (CNPq) e ao Programa de Apoio ao Desenvolvimento Científico da Faculdade de Ciências Farmacêuticas da Unesp (PADC - FCF UNESP), pelo apoio a nossos projetos. 


\section{REFERÊNCIAS}

1. Ribeiro ALA. Resolução RDC № 33 / ANVISA/MS: uma análise crítica do roteiro de inspeção para farmácias com manipulação [Dissertação]. Niterói; RJ: Universidade Federal Fluminense; 2003.

2. Silva RF da, Nascimento Filho AP, Mendonça DC. Estratégias competitivas no mercado farmacêutico brasileiro: uma abordagem sobre o setor magistral. Trabalho apresentado ao 8‥ SIMPEP. Bauru, Brasil; 2006.

3. Agência Nacional de Vigilância Sanitária - ANVISA. Resolução RDC № 33, de 19 de abril de 2000. Brasília (DF): Diário Oficial [da] República Federativa do Brasil; 19 abr. 2003.

4. Agência Nacional de Vigilância Sanitária - ANVISA] Resolução RDC № 354, de 18 de dezembro de 2003. Brasília (DF): Diário Oficial [da] República Federativa do Brasil; 22 dez. 2003.

5. Agência Nacional de Vigilância Sanitária - ANVISA. Subsídios à discussão sobre a proposta de regulamentação para farmácias magistrais. Rev. Saúde Publ. 2005;39(4):42-6.

6. Agência Nacional de Vigilância Sanitária - ANVISA. Resolução RDC № 214, de 12 de dezembro de 2006. Brasília (DF): Diário Oficial [da] República Federativa do Brasil; 18 dez. 2006.

7. Agência Nacional de Vigilância Sanitária - ANVISA. Resolução RDC № 67, de 8 de outubro de 2007. Brasília (DF): Diário Oficial [da] República Federativa do Brasil; 9 out. 2007.

8. Helou JH, Cimino JS, Daffre C. Farmacotécnica. São Paulo: Artpress; 1975.

9. Ansel HC, Popovich NG, Allen Júnior L. Farmacotécnica. Formas farmacêuticas e sistemas de liberação de fármacos. $6^{a}$ ed. São Paulo: Editorial Premier; 2000.

10. Prista LN, Alves AC, Morgado R, Lobo JS. Tecnologia farmacêutica. $6^{a}$ ed. Lisboa: Fundação Calouste Gulbenkian; 2003.

11. Conselho Federal de Farmácia - CFF. Resolução № 357, de 20 de abril de 2001. Brasília (DF): Diário Oficial [da] República Federativa do Brasil; 20 abr. 2001.

12. Miguel MD, Zanin SMW, Miguel OG, Roze AO, Oyakawa CN, Oliveira ABO. Cotidiano das farmácias de manipulação. Visão Acadêmica 2002;3(2):103-8.

13. Brasil. Política Nacional de Medicamentos, série $C$, projetos, programas e relatórios, n. 25. Brasília (DF); 2001.

14. Rezende AJ, Pereira CA, Athayde TR, Leite Filho GA. Análise do comportamento dos preços de medicamentos na cidade de São Paulo. Trabalho apresentado ao $8^{\text {o }}$ Congreso del Instituto Internacional de Costos. Punta del Este, Uruguay; 2003. 
Revista Baiana de Saúde Pública

15. Pereira AC, Servilieri KM. Um estudo de caso sobre a mensuração dos custos em uma farmácia de manipulação. In: Anais do 9o Congresso Internacional de Custos. Florianópolis, SC; 2005.

16. Barros JAC. A (des)informação sobre medicamentos: o duplo padrão de conduta das empresas farmacêuticas. Cad. Saúde Publ. 2000;16(2):421-7.

17. Krupka LR, Vener AM. Prescription drug advertising: trends and implications. Soc. Sci. Med. 1985;20(3):191-7.

18. Barros JAC. Estratégias mercadológicas da indústria farmacêutica e o consumo de medicamentos. Rev. Saúde Publ. 1983;17(5):377-86.

19. Fagundes MJD, Soares MGA, Diniz NM, Pires JR, Garrafa V. Análise bioética da propaganda e publicidade de medicamentos. Ci. Saude Col. 2007;12(1):221-9.

20. Pizzol FD, Silva, T, Schenkel EP. Análise da adequação das propagandas de medicamentos dirigidas à categoria médica distribuídas no Sul do Brasil. Cad. Saúde Publ. 1998;14(1):85-91.

21. Fiaschetti MA. Opiniões e atitudes dos médicos frente às ações promocionais da indústria farmacêutica [Dissertação]. Araraquara: Faculdade de Ciências Farmacêuticas; 2007.

22. Agência Nacional de Vigilância Sanitária - ANVISA. Resolução RDC № 96, de 17 de dezembro de 2008. Brasília (DF): Diário Oficial [da] República Federativa do Brasil, 18 dez. 2008.

23. Martinelli HK, Castellani AM, Gonçalves JE, Gonçalves RAC. Avaliação do controle de qualidade realizado nas farmácias de manipulação e homeopáticas de Maringá, Estado do Paraná. Acta Sci. Health Sci. 2005;27(2):137-43.

24. Agência Nacional de Vigilância Sanitária - ANVISA. Laboratórios Analíticos em Saúde. Extraído de [http://www.anvisa.gov.br/reblas/apresentacao.htm], acesso em [13 de setembro de 2009].

25. Rumel D, Nishioka SA, Santos AAM dos. Intercambialidade de medicamentos: abordagem clínica e o ponto de vista do consumidor. Rev. Saude Publ. 2006;40(5):921-7.

26. Agência Nacional de Vigilância Sanitária - ANVISA. Resolução RDC № 135, de 29 de maio de 2003. Diário Oficial [da] República Federativa do Brasil. Brasília, DF; 20032 jun. 
27. Posição da Escola Nacional de Saúde Pública Sérgio Arouca (ENSP/ FIOCRUZ), do Instituto Nacional de Controle de qualidade em Saúde (INCQS/FIOCRUZ), da Escola Politécnica de Saúde Joaquim Venâncio (EPSJV/FIOCRUZ) e da Fundação Oswaldo Cruz (FIOCRUZ) em relação à consulta pública da ANVISA N 31/2005, sobre o regulamento das Boas Práticas de manipulação de medicamentos em farmácias. Extraído de [http://www4.ensp.fiocruz.br/eventos_novo/ dados/arq533.pdf.], acesso em [15 de setembro de 2009].

Recebido em 24.2.2010 e aprovado em 23.9.2010. 